Please do not remove this page

RMIT

UNIVERSITY

\title{
Synthetic cannabis: A comparison of patterns of use and effect profile with natural cannabis in a large global sample
}

Winstock, Adam; Barratt, Monica

https://researchrepository.rmit.edu.au/esploro/outputs/9921861300301341/filesAndLinks?institution=61RMIT_INST\&index=null

Winstock, A., \& Barratt, M. (2013). Synthetic cannabis: A comparison of patterns of use and effect profile with natural cannabis in a large global sample. Drug and Alcohol Dependence, 131(1-3), 106-111.

https://doi.org/10.1016/j.drugalcdep.2012.12.011

Document Version: Accepted Manuscript

Published Version: https://doi.org/10.1016/j.drugalcdep.2012.12.011

Repository homepage: https://researchrepository.rmit.edu.au

CC BY-NC-ND V4.0

(C) 2013 Published by Elsevier Ireland Ltd.

Downloaded On 2023/04/26 18:51:21 +1000

Please do not remove this page 
Synthetic cannabis: a comparison of patterns of use and effect profile with natural cannabis in a large global sample

Adam R. Winstock ${ }^{1,2}$

Monica J. Barratt ${ }^{3}$

${ }^{1}$ South London and Maudsley NHS Trust / Kings College London

${ }^{2}$ Global Drug Survey, London, United Kingdom.

${ }^{3}$ National Drug Research Institute, Curtin University, Melbourne, Australia.

Word count: 4272 


\section{Abstract}

Background: The last decade has seen the appearance of myriad novel psychoactive substances with diverse effect profiles. Synthetic cannabinoids are among the most recently identified but least researched of these substances.

Methods: An anonymous online survey was conducted in 2011 using a quantitative structured research tool. Missing data (median 2\%) were treated by available-case analysis.

Results: Of 14,966 participants, 2,513 (17\%) reported use of synthetic cannabis. Of these, $980(41 \%$ of 2,417$)$ reported its use in the last 12 months. Almost all recent synthetic cannabis users (99\% of 975 ) reported ever use of natural cannabis. Synthetic cannabis reportedly had both a shorter duration of action $(z=17.82, p<.001)$ and quicker time to peak onset of effect $(z=-9.44, p<.001)$ than natural cannabis. Natural cannabis was preferred to synthetic cannabis by $93 \%$ of users, with natural cannabis rated as having greater pleasurable effects when high $(t(930)=-37.1, p<.001, d=-$ $1.22)$ and being more able to function after use $(t(884)=-13.3, p<.001, d=-0.45)$. Synthetic cannabis was associated with more negative effects $(t(859)=18.7, p<.001, d$ $=0.64)$, hangover effects $(t(854)=6.45, p<.001, d=0.22)$ and greater paranoia $(t(889)$ $=7.91, p<.001, d=0.27$ ).

Conclusions: Users report a strong preference for natural over synthetic cannabis. The latter has a less desirable effect profile. Further research is required to determine longer term consequences of use and comparative dependence potential.

Key words: Synthetic cannabinoids, Cannabis, Epidemiology, Online survey (Abstract word count: 250) 


\section{Introduction}

Synthetic cannabinoid receptor agonists (hereon 'synthetic cannabinoids') produce subjective effects similar to cannabis in humans, but are structurally dissimilar from $\Delta$ 9-tetrahydrocannabinol (THC), the principal cannabinoid found in cannabis (Fattore and Fratta, 2011; Hudson and Ramsey, 2011; Vardakou et al., 2010). Synthetic cannabinoid products were first reported in 2004, with initial increased use reported in 2008 in Europe (European Monitoring Centre for Drugs and Drug Addiction, 2010) and in 2010-11 in the US (Wells and Ott, 2011), Australia (Warhaft, 2011) and New Zealand (Schep et al., 2011). The products are typically manufactured by applying synthetic cannabinoids onto relatively inert plant matter that can be smoked (Fattore and Fratta, 2011; Hudson and Ramsey, 2011; Vardakou et al., 2010). Smokable synthetic cannabinoid products have been sold under several commercial brands, with Spice the most common in Europe, K2 in the US, and Kronic in Australia and New Zealand.

There are a large number of different synthetic cannabinoid chemicals, many of which have not yet been formally identified in synthetic cannabis products. There has been growing recognition of the harms associated with synthetic cannabinoid intoxication, including: tachycardia, psychosis, agitation/irritation, panic/anxiety, vomiting, and seizures (Castellanos and Thornton, 2012; Fattore and Fratta, 2011; Hoyte et al., 2012; Wells and Ott, 2011), and there have been some reports of dependence and withdrawal after prolonged use (Every-Palmer, 2011; Vandrey et al., 2012; Zimmermann et al., 2009). It can be argued that the harm profile of synthetic cannabis products is greater than the harm profile of natural cannabis, especially in relation to seizures as cannabis is actually an anti-convulsant (de Havenon et al., 2011; Hoyte et al., 2012; Schneir and Baumbacher, 2012).

Due to their structural dissimilarity to cannabis, synthetic cannabinoid products were legal when they first became popular. Their use was also difficult to monitor because standards to detect their metabolites in urine and blood tests were still under development (See de Jager et al., 2012; Möller et al., 2011; Sobolevsky et al., 2010). In response to increased prevalence of use and a growing harm profile, prohibitions of synthetic cannabinoids have now been enacted in a number of countries including, but not limited to, the US (United Nations Office on Drugs and Crime, 2011), UK (Advisory 
Council on the Misuse of Drugs, 2009), New Zealand (Brown, 2011) and Australia (Therapeutic Goods Administration (TGA), 2012). In many cases, prohibitions have not stopped the sale of synthetic cannabis products. Instead, manufacturers have produced products with new formulas containing as-yet-unscheduled psychoactive components (Dargan et al., 2011; Fattore and Fratta, 2011; Hammersley, 2010; Rosenbaum et al., 2012). Other models of control, including consumer safety or medicines legislation, have been proposed (Evans-Brown et al., 2011; Hughes and Winstock, 2012).

To date, there have been two published survey of synthetic cannabinoids users recruited from the general population (not in treatment) (Barratt et al., 2012, with an Australian sample of 316; Vandrey et al., 2012, with an international sample of 168). In both studies, the samples were mostly male, were aged in their mid-twenties, and were mostly either employed or studying. In the Vandrey study, no respondent reported ever seeking or receiving treatment for problems associated with synthetic cannabinoid use, and in the Barratt study, four respondents reported seeking help after their last session of synthetic cannabis use. In both studies, most respondents were also cannabis users (84\% in Vandrey's sample and 96\% in Barratt's sample).

The only other studies reporting on large (100+) samples of synthetic cannabis users have used health administrative records of calls to poisons centres in the USA (Hoyte et al., 2012; but also see Fernandez et al., 2011; Forrester et al., 2011) and Sweden (Westerbergh and Hultèn, 2011). In the largest of these studies, 1,353 single-agent exposures to synthetic cannabinoids were reported in the US National Poison Data System over a 9-month period in 2010 (Hoyte et al., 2012). The median age of exposure was 20 years (interquartile range [IQR] 17-25) and 74\% were male. Only $7.3 \%$ of exposures were coded as potentially life threatening, and 1 death of a 54-year-old man from cardiac arrest was recorded. In $78.4 \%$ of cases clinical effects lasted 8 hours or less.

No studies have yet been published that explore the relationship between synthetic and natural cannabis use patterns and assess users' perceptions of synthetic cannabinoids in comparison to 'natural' cannabis preparations. It is important to understand these patterns and perceptions because synthetic cannabis may be used as a substitute for cannabis, especially for those who are subject to drug testing in their workplace or due to participation in drug treatment or the justice system (Castellanos et al., 2011; Every- 
Palmer, 2011; Schifano et al., 2009; Vandrey et al., 2012). In this paper we present the largest self-report study to date describing the demographic and drug use characteristics of recent synthetic cannabinoid users, and their perceptions of and comparisons between important attributes of both synthetic and natural cannabis.

\section{Method}

\subsection{Design}

An anonymous, online survey of drug and alcohol use was designed and conducted by Global Drug Survey (www.globaldrugsurvey.com/mixmag2012). It was promoted in partnership with the dance music magazine Mixmag and the Guardian newspaper. The survey was widely promoted through our media partner websites and social networking media such as Facebook and Twitter. The research tool was based on previous work done by the research group. Further information about the utility, validity, and limitations of the current methodology has been discussed elsewhere (McCambridge et al., 2007; Winstock et al., 2001; Winstock et al., 2012; Winstock et al., 2011b; Winstock et al., 2002). Ethical approval was received from the Joint South London and Maudsley and Institute of Psychiatry NHS Research Ethics Committee.

\subsection{Measures}

Demographics and prevalence of lifetime (ever used) and recent use (last year and number of days in the last month) of a large number of substances including synthetic cannabinoids (synthetic cannabis) were collected. In addition to prevalence data on the use of synthetic cannabis we also sought the effect profile of synthetic cannabis compared to that of herbal/natural cannabis across a range of acute positive and negative global and specific effect domains (rated 0-10) (nature of sleep after use, ability to function after use, memory impairment, increase in appetite, paranoia, pleasurable effects when high, negative effects when high, hangover effect). We also sought an overall preference and a comparison of individual user perception of the consistency of the product, its value for money, harmful effects on the lungs and addictiveness perception using the same scale. In addition we assessed comparison in the time to onset of effect, the time to peak effect and the duration of effect after use by the smoking route. The use of the effect profile questions 'pleasurable high' of the drug, 
the 'negative effects of the drug when high' and value for money have previously been used in the risk assessment of mephedrone carried out by this group (Winstock et al., $2011 b)$. Intentions to reduce or cease use and demand for treatment were also assessed.

\subsection{Analysis}

Due to the sensitive and incriminating nature of the information collected, no IP addresses or other potentially identifying information was collected from responders and therefore it was impossible to eliminate multiple entries from the same IP address. The dataset was scanned for identical entries but none were found. We consider it unlikely that anyone would undertake to complete the survey more than once as this would entail large amounts of time for no obvious gain as there were no material incentives (e.g., lotteries, prizes, payments) offered.

Most questions in this survey generated some missing responses. Of the 87 variables or comparisons reported in this paper, there was a median of $2 \%$ missing data (Interquartile range 1\%-4\%, range $0 \%-14 \%$ ). Due to the relatively low level of missing data, we used available-case analysis or case-wise deletion (including all cases with observed values on the variables included in any particular analysis). Excluding all cases with any missing data (complete-case analysis or list-wise deletion) would unnecessarily reduce our sample size, and more complex imputation or modelling is not usually justified if the proportion of missing data is minimal (Penny \& Atkinson, 2012). We have noted the valid total $N$ after each statistic.

The analysis primarily involved descriptive statistics. Medians with IQR were reported as these statistics are less biased descriptors of count variables. Wilcoxon matchedpairs signed-ranks test were conducted to compare ordinal responses for speed of onset, time to peak effect and duration of peak effect of synthetic versus natural cannabis. Dependent samples $t$-tests were conducted to compare self-rated effects of synthetic and natural cannabis, with the magnitude of the difference measured using Cohen's $d$. Wilcoxon rank-sum tests were used to compare the median days of use by (a) desire to cut down and (b) interest in help or treatment, for both synthetic and natural cannabis.

The variable 'years since first use' was calculated by subtracting age of first use from current age then adding 1 . A few respondents reported an age of first use older than 
their current age $(n=4)$ and some $(n=27)$ reported improbable or impossible ages of first use for synthetic cannabis, which has only been reported since 2004. In these 31 cases, age of first use and years since first use of synthetic cannabis were recoded to missing. Age of first use for natural cannabis was checked in the same way but no improbable or impossible values were found. All analyses were conducted using Stata 11 (College Station, TX).

\section{Results}

Between November 23 and December 21, 2011, a total of 15,200 responses were received from around the world, with $98 \%(N=14,855)$ providing their country of origin. Of these, just over half $(52.0 \%, n=7,719)$ were from the UK and just under one quarter $(22.6 \%, n=3,362)$ were from the USA. Other significant nation contributors to the sample included Canada $(5.5 \%, n=815)$, Australia $(2.8 \%, n=413)$ and Ireland $(2.0 \%, n=302)$. Over two thirds $(70.7 \%$ of 14,923$)$ were male, the median age was 26 years (IQR 22-33, $N=14,889)$, and almost all $(91.5 \%$ of 14,701$)$ identified their ethnicity as White.

\subsection{Demographic profile of synthetic cannabis users}

Of the 14,966 participants who responded, 2,513 (16.8\%) reported ever having used synthetic cannabis. Of these, $980(40.6 \%$ of 2,417$)$ reported use of synthetic cannabis in the last 12 months, hereon described as recent synthetic cannabis users. Recent users were predominantly male (79.6\% of 953) with a median age of 23 years (IQR 19-28) and mean age of 25.3 years ( $S D 8.32$, range 18-65, $N=961$ ). Two-thirds reported currently working (66.6\%), over half reported currently studying (53.3\%), and only $8.8 \%$ were currently neither working nor studying $(N=931)$. One third $(36.4 \%)$ of the sample had completed a university degree $(N=935)$. The respondents commonly lived with parents $(29.2 \%)$, friends $(28.2 \%)$ or a partner $(21.6 \%)$ while $14.1 \%$ reported living alone (7.0\% other, $N=964)$. Most reported their ethnicity as White $(90.8 \%, N=$ $944)$ and their sexual orientation as heterosexual $(82.3 \%, N=932)$. Half the sample $(49.3 \%, n=471)$ were from the US and a quarter reporting living in the UK $(26.6 \%, n=$ 254). Other countries of origin which were reported by 10 or more respondents included: Australia (5.6\%, $n=53)$, New Zealand (3.5\%, $n=33)$, Canada (1.8\%, $n=17)$, 
Finland $(1.8 \%, n=17)$ and Hungary $(1.3 \%, n=12)$. The remaining 98 respondents $(10.3 \%)$ came from around the world with countries contributing 5 or more participants including Japan, Ireland, Mexico, Poland and South Africa $(N=955)$.

\subsection{Patterns of synthetic cannabis use}

Among recent users, the median age of first use of synthetic cannabis was 21 years (IQR 18-26, range $13-65, N=897)$. Consistent with the emergent status of this drug, the median number of years since first use was 2 (IQR $1-3$, range $1-8, N=883$ ). The majority of last-12-month synthetic cannabis users $(60.6 \%, N=904)$ did not report use of the drug within the last 30 days. Of the 356 who did report last month use, the median days of use in the past month was 3 (IQR 1-8). One third (32.6\%) reported use on only one occasion, over one third (36.8\%) reported use on 5 or more occasions (more than weekly), and 17 (4.8\%) reported daily use $(N=356)$.

\subsection{Patterns of 'natural' cannabis use among recent synthetic cannabis users}

Almost all recent synthetic cannabis users (99.3\%, $N=975)$ reported ever use of some form of natural cannabis, including 95.9\% reporting ever use of cannabis 'grass' $(N=$ 966), 83.5\% reporting ever use of cannabis 'skunk' ( $N=964)$ and $80.5 \%$ reporting ever use of cannabis 'resin' $(N=969)$. The median age of first use of any form of natural cannabis was 16 years (IQR $14-18$, range $12-30, N=942$ ) and the median number of years since first use was 7 (IQR 4-12, range 1-45, $N=927$ ). Use of any form of natural cannabis in the last 12 months was reported by $95.0 \%$ of recent synthetic cannabis users $(N=960)$, including $82.7 \%$ reporting recent use of cannabis 'grass', $72.0 \%$ reporting recent use of cannabis 'skunk', and 59.9\% reporting recent use of cannabis 'resin'. Of those who reported any natural cannabis use in the last 12 months, most $(88.4 \%)$ also reported use in the last month $(N=905)$. Of the 800 who reported last month use of any form of natural cannabis, the median days of use in the past month was 17 (IQR 6-29), 6.6\% reported use on only one occasion, 81.1\% reported use on 5 or more occasions (more than weekly), and $24.6 \%$ reported daily use.

Figure 1 illustrates the frequency of synthetic and natural cannabis use among recent users of both drug types $(N=837)$, with ' 0 ' representing 'not in the last month' and each number representing the number of days used in the last month. The most common pattern of use was last month cannabis users (days $>0$ ) who reported use of synthetic 
cannabis 'not in the last month' (days $=0)(52.1 \%)$, whereas only $2.7 \%$ reported last month synthetic cannabis use (days $>0$ ) with no last month natural cannabis use (days $=0$ ). Most of the sample (79.5\%) reported a greater number of days using cannabis than synthetic cannabis, $12.3 \%$ reported the same number of days, and $8.2 \%$ reported a greater number of days using synthetic cannabis than natural cannabis. Only 7 (0.84\%) respondents reported daily use of both types of cannabis. Figure 1 indicates that natural cannabis was used more regularly and recently than synthetic cannabis among this sample.

Insert Figure 1 about here

\subsection{Other drug use}

Prevalence in the last year and month and the number of days used per month for drugs other than synthetic cannabis are listed in Table 1. Most recent synthetic cannabis users consumed alcohol, cannabis, tobacco and energy drinks in the last month (see Table 1). Over one third of the respondents were daily tobacco smokers $(352,36.2 \%$ of 972) whereas daily alcohol consumption was only rarely reported (30,3.1\% of 970). Around half of this sample reported consumption of MDMA in the last year and about one third reported consumption of mushrooms, cocaine, LSD and/or benzodiazepines in the last year (see Table 1).

Insert Table 1 about here

\subsection{Comparison of effects between synthetic cannabis and natural cannabis}

Participants were asked to compare the speed of onset, time to peak effect and duration of effect of synthetic and 'natural/herbal' cannabis when smoked (see Figure 2). The reported speed of onset for synthetic and natural cannabis was almost the same, with about $60 \%$ of the sample reporting onset within 5 minutes, a quarter reporting 6-10 minutes, and the remainder more than 10 minutes (Wilcoxon signed-rank test: $z=1.11$, 
$p=.268$, see Figure 2A). Synthetic cannabis was reported to have a shorter time until peak effect when compared with natural cannabis: over half of the sample reported the peak effect occurred within 5 minutes with synthetic cannabis whereas the most common response for natural cannabis was 6-10 minutes until peak effect (Wilcoxon signed-rank test: $z=-9.44, p<.001$, see Figure 2B). Synthetic cannabis was also reported to have a significantly shorter duration of action than natural cannabis: two thirds of the sample reported only feeling 'stoned' for one hour after smoking a typical joint/bong of synthetic cannabis, whereas three quarters of the sample reported feeling the effects of natural cannabis for 2 hours or longer (Wilcoxon signed-rank test: $z=-17.82, p<.001$, see Figure $2 \mathrm{C}$ ).

Insert Figure 2 about here

In addition to the comparison in the onset and duration of effect participants were asked to compare their experience of synthetic cannabis with that of smoked natural cannabis across 12 broad positive and negative effect domains and rate each of these on a scale of $1-10$ (1=low $10=$ high, see Table 2$)$. Natural cannabis was rated as having greater pleasurable effects when high and resulting in greater increases in appetite than synthetic cannabis (both $t$-tests resulted in large effect sizes). Although natural cannabis was reported to have greater sedating effects, participants paradoxically reported being more able to function after natural cannabis compared with synthetic cannabis $(t$-tests resulted in medium effect sizes). Natural cannabis was reported to produce more memory impairment, be more addictive, and be more consistent as a product than synthetic cannabis, but these comparisons produced only small effect sizes. Natural cannabis was reported to represent better value for money. Synthetic cannabis was reported to produce more negative effects when high and more harmful effects on the lungs than natural cannabis ( $t$-tests resulted in medium effect sizes). Synthetic cannabis was also associated with more hangover effects and greater paranoia than natural cannabis, although these comparisons produced only small effect sizes. 
Those reporting a preference of synthetic cannabis over natural cannabis were asked which of four reasons was the most important in forming their preference. Of the 69 (7.2\% of 956) respondents who expressed a preference for synthetic cannabis, the most important reason was effect $(40,58.0 \%)$, availability $(13,18.9 \%)$, not as easily detected in urine screens $(10,14.5 \%)$ and cost $(6,8.7 \%)$.

\subsection{Demand for treatment}

Seven per cent (64 of 980) reported that they would like to use less synthetic cannabis in the coming year and $18(1.8 \%)$ reported that they would like help to reduce or cease their use of synthetic cannabis. Of the 356 who reported last month use, those who reported that they would like to use less synthetic cannabis were using it on significantly more days (median 6 days in the last 30, IQR 2-24, $n=32$ ) than other last month users (median 2 days, IQR 1-7, $n=324 ; z=-3.25, p=.001$ ). Similarly, of the 356 who reported last month use, those who reported that they would like help to reduce their use were using it significantly more often (median 27 days in the last 30, IQR 7-30, $n=12$ ) than other last month users (median 2 days, IQR 1-7, $n=344 ; z=-3.87, p<$ $.001)$.

In contrast, $17 \%$ of recent cannabis users (167 of 968) reported that they would like to use less natural cannabis in the coming year and 37 (3.8\%) reported that they would like help to reduce or cease their use of natural cannabis. Of the 800 who reported last month use of cannabis, those who reported that they would like to use less were using it on significantly more days (median 23 days in the last 30, IQR 10-30, $n=153$ ) than other last month users (median 15 days, IQR 5-28, $n=647 ; z=-4.15, p<.001$ ). Similarly, those who reported that they would like help to reduce their use were using it significantly more often (median 25 days, IQR 15-30, $n=35$ ) than other last month users (median 16 days, IQR 6-29, $n=765 ; z=-2.41, p=.016$ ). 


\section{Discussion}

This is the largest survey of synthetic cannabinoid users ever conducted. The findings suggest that when smoked users are able to differentiate between synthetic and natural cannabis in terms of effect profile with a clear preference for natural cannabis over synthetic cannabis by 93\%. Synthetic cannabis showed significantly higher scores for self-reported hangover effects and negative effects when high compared with natural cannabis, while natural cannabis scored significantly higher in terms of pleasurable effects when high and ability to function while high.

In keeping with these findings natural cannabis was used more frequently and recently than synthetic cannabis. Only a small minority of users appeared to have fully substituted natural for synthetic. This suggests that in terms of the public health consequences of use cannabis remains a far more significant issue.

What remains unexplained is why-despite wide availability of natural cannabis and clear preference for its effects over synthetic products - there is still a demand for a 'legal' cannabis-like product. While the attraction for novel psychoactive drugs such as mephedrone could be understood in the context of decline in the purity of traditional stimulants (Winstock et al., 2010; Winstock and Ramsey, 2010), this does not appear to be the case with cannabis. In their Australian study, Barratt et al. (2012) found that among a sample of mainly cannabis users, the second most commonly mentioned reason for first trying synthetic cannabis was its legal status. It may be the case that cannabis users desire a legal cannabis-like alternative so they can avoid problems often associated with cannabis prohibition, like stigma, arrest, paranoia, problems with police, and confiscation of drugs.

Among the minority expressing a preference for the effects of synthetic products, its reduced chance of detection in urine drug screens may not be representative of other populations of users such as those who may be urine tested for work or in correctional facilities. Whether or not these products will be attractive to a large number of cannabis naïve users remains worthy of on-going monitoring.

The wide variation in potency and composition between synthetic cannabis is a particular risk facing users, though surprisingly, the perceived comparison in product consistency between synthetic and natural cannabis was not as large as may have been 
expected. Whether or not this is because manufactures are aware of the disparate potency of different synthetic cannabinoids and titrate their addition to herbal blends accordingly is uncertain.

\subsection{Limitations}

The study although of a good size is limited by the self-nominating nature of the sample and the self-report methodology. These limitations and others have been discussed more fully elsewhere (McCambridge et al., 2005a; McCambridge et al., 2005b; Winstock et al., 2001; Winstock et al., 2011b; Winstock et al., 2002). The study was accessed from widely divergent internet sources, with only just over one thirds of participants accessing the survey through one of our media partners. While our previous studies have focused on accessing clubbers this current sample reflect a far broader group with only 362 (36.9\%) reporting they go clubbing monthly or more often. 296 (30.2\%) report that they never go clubbing.

We have previously shown that self-report studies among dance drug using populations may be a valid and effective tool for describing the effect profile of novel drugs and detecting the appearance of new drugs (Winstock et al., 2011a; Winstock et al., 2002). We accept that when judged against traditional epidemiological criteria for a good public health surveillance system this method has significant limitations, not least because it recruits from a self-nominating population that is relatively poorly characterised. In addition it relies upon population self-reported experiences with a substance whose true composition is uncertain. High levels of poly-drug use, confounding effects from other substances and recall bias are all significant issues. It is also very possible that the effect profile with synthetic cannabinoids will change with repeated episodes of use and with differing routes of administration (e.g., oral). However in the early stages of the appearance of a new drug, approaches such as these are often the only initial ways of accessing data rapidly and efficiently. We believe our approaches can usefully guide future research as well as inform those who choose to use novel substances.

\subsection{Future research}

As with all novel psychoactive drugs on-going research is required to monitor the evolution of harms and patterns of use. Triangulation of data from health services and 
law enforcement, basic scientists and users is required to support the development and implementation of optimal public health strategies to address the appearance of these drugs. Particular attention will be required to monitor whether there are any longer term neurotoxic or behavioural harms consequent upon repeated exposure.

\subsection{Conclusion}

Synthetic cannabinoids represent a diverse group of potent psychoactive compounds that can substitute for natural cannabis. Although the pattern of use reported in this study and the comparatively low reported demand for treatment may reassure policy makers and health providers it is too early to confidently predict that its currently low level of uptake and perceived attractiveness will limit its wider use and the experience of harms. Perhaps the most effective public health approach to addressing a class of compounds which are likely to remain widely available is publicity across the less desirable effect profile and risks of increased anxiety and agitation associated with synthetic cannabinoid use. 
References

Advisory Council on the Misuse of Drugs, 2009. Consideration of the major cannabinoid agonists. Home Office, London.

Barratt, M. J., Cakic, V., Lenton, S., 2012. Patterns of synthetic cannabinoid use in Australia. Drug Alcohol Rev, Early View. doi: 10.1111/j.1465-3362.2012.00519.x

Brown, K., 2011. New Zealand bans synthetic cannabinoids. BMJ 343, d5395.

Castellanos, D., Singh, S., Thornton, G., Avila, M., Moreno, A., 2011. Synthetic cannabinoid use: A case series of adolescents. J Adolesc Health 49, 347-349.

Castellanos, D., Thornton, G., 2012. Synthetic cannabinoid use: Recognition and management. J Psychiatr Pract 18, 86-93.

Dargan, P.I., Hudson, S., Ramsey, J., Wood, D.M., 2011. The impact of changes in UK classification of the synthetic cannabinoid receptor agonists in 'Spice'. Int J Drug Policy 22, 274-277.

de Havenon, A., Chin, B., Thomas, K.C., Afra, P., 2011. The secret "spice": An undetectable toxic cause of seizure. The Neurohospitalist 1, 182-186.

de Jager, A.D., Warner, J.V., Henman, M., Ferguson, W., Hall, A., 2012. LC-MS/MS method for the quantitation of metabolites of eight commonly-used synthetic cannabinoids in human urine - An Australian perspective. J Chromatogr B 897, 22-31.

European Monitoring Centre for Drugs and Drug Addiction, 2010. 2010 Annual report on the state of the drugs problem in Europe. EMCDDA, Luxembourg.

Evans-Brown, M., Bellis, M.A., McVeigh, J., 2011. Should “legal highs” be regulated as medicinal products? BMJ 342, 501.

Every-Palmer, S., 2011. Synthetic cannabinoid JWH-018 and psychosis: an explorative study. Drug Alcohol Depend 117, 152-157.

Fattore, L., Fratta, W., 2011. Beyond THC: the new generation of cannabinoid designer drugs. Frontiers in Behavioral Neuroscience 5, Article 60.

Fernandez, M.C., Roth, B., Villareal, C.L., 2011. Fake marijuana causing real problems in Texas. Clin Toxicol 49, 212.

Forrester, M.B., Kleinschmidt, K., Schwarz, E., Young, A., 2011. Synthetic cannabinoid exposures reported to Texas Poison Centers. J Addict Dis 30, 351-358.

Hammersley, R., 2010. Dangers of banning spice and the synthetic cannabinoid agonists. Addiction 105, 373.

Hoyte, C.O., Jacob, J., Monte, A.A., Al-Jumaan, M., Bronstein, A.C., Heard, K.J., 2012. A characterization of synthetic cannabinoid exposures reported to the National Poison Data System in 2010. Ann Emerg Med 60, 435-438.

Hudson, S., Ramsey, J., 2011. The emergence and analysis of synthetic cannabinoids. Drug Test Anal 3, 466-478.

Hughes, B., Winstock, A.R., 2012. Controlling new drugs under marketing regulations. Addiction 107, 1894-1899.

McCambridge, J., Hunt, N., Winstock, A., Mitcheson, L., 2005a. Has there been a decline in the prevalence of cannabis use among British nightclubbers? Five-year survey data. Drug Educ Prev Policy 12, 167-169.

McCambridge, J., Mitcheson, L., Winstock, A., Hunt, N., 2005b. Five-year trends in patterns of drug use among people who use stimulants in dance contexts in the United Kingdom. Addiction 100, 1140-1149. 
McCambridge, J., Winstock, A., Hunt, N., Mitcheson, L., 2007. 5-Year trends in use of hallucinogens and other adjunct drugs among UK dance drug users. Eur Addict Res 13, 57-64.

Möller, I., Wintermeyer, A., Bender, K., Jübner, M., Thomas, A., Krug, O., Schänzer, W., Thevis, M., 2011. Screening for the synthetic cannabinoid JWH-018 and its major metabolites in human doping controls. Drug Test Anal 3, 609-620 .

Penny, K.I., Atkinson, I., (2012). Approaches for dealing with missing data in health care studies. J Clin Nurs 21, 2722-2729.

Rosenbaum, C., Carreiro, S., Babu, K., 2012. Here today, gone tomorrow ...and back again? A review of herbal marijuana alternatives (K2, Spice), synthetic cathinones (bath salts), kratom, salvia divinorum, methoxetamine, and piperazines. J Med Toxicol 8, 15-32.

Schep, L.J., Slaughter, R.J., Temple, W.A., Nair, S.M., Gee, P., 2011. Synthetic cannabinoid analogues-not repeating past mistakes. N Z Med J 124, 85-86.

Schifano, F., Corazza, O., Deluca, P., Davey, Z., Di Furia, L., Farre, M., Flesland, L., Mannonen, M., Pagani, S., Peltoniemi, T., Pezzolesi, C., Scherbaum, N., Siemann, H., Skutle, A., Torrens, M., Van Der Kreeft, P., 2009. Psychoactive drug or mystical incense? Overview of the online available information on Spice products. Int J Cult Ment Health 2, 137-144.

Schneir, A.B., Baumbacher, T., 2012. Convulsions associated with the use of a synthetic cannabinoid product. J Med Toxicol 8, 62-64.

Sobolevsky, T., Prasolov, I., Rodchenkov, G., 2010. Detection of JWH-018 metabolites in smoking mixture post-administration urine. Forensic Sci Int 200, 141-147.

Therapeutic Goods Administration (TGA), 2012. Final decisions and reasons for decisions by delegates of the secretary to the Department of Health and Ageing. Commonwealth of Australia, Canberra.

United Nations Office on Drugs and Crime, 2011. Synthetic cannabinoids in herbal products. United Nations Office on Drugs and Crime, Vienna.

Vandrey, R., Dunn, K.E., Fry, J.A., Girling, E.R., 2012. A survey study to characterize use of Spice products (synthetic cannabinoids). Drug Alcohol Depend 120, 238-241.

Vardakou, I., Pistos, C., Spiliopoulou, C., 2010. Spice drugs as a new trend: Mode of action, identification and legislation. Toxicol Lett 197, 157-162.

Warhaft, G., 2011. Not for human consumption? The banning of synthetic cannabinoids. Of Substance 9, 14-17.

Wells, D.L., Ott, C.A., 2011. The "new" marijuana. Ann Pharmacother 45, 414-417.

Westerbergh, J., Hultèn, P., 2011. Novel synthetic cannabinoids, CRA13, JWH-015, JWH081 and JWH-210 - Detected in a case series. Clin Toxicol 49, 222.

Winstock, A., Mitcheson, L., Ramsey, J., Davies, S., Puchnarewicz, M., Marsden, J., 2011a. Mephedrone: use, subjective effects and health risks. Addiction 106, 1991-1996.

Winstock, A.R., Griffiths, P., Stewart, D., 2001. Drugs and the dance music scene: A survey of current drug use patterns among a sample of dance music enthusiasts in the UK. Drug Alcohol Depend 64, 9-17.

Winstock, A.R., Marsden, J., Mitcheson, L., 2010. What should be done about mephedrone? BMJ 340, 1605.

Winstock, A.R., Mitcheson, L., Gillatt, D.A., Cottrell, A.M., 2012. The prevalence and natural history of urinary symptoms among recreational ketamine users. BJU Int 110, E164-E165.

Winstock, A.R., Mitcheson, L.R., Deluca, P., Davey, Z., Corazza, O., Schifano, F., 2011b. Mephedrone, new kid for the chop? Addiction 106, 154-161. 
Winstock, A.R., Ramsey, J.D., 2010. Legal highs and the challenges for policy makers. Addiction 105, 1685-1687.

Winstock, A.R., Wolff, K., Ramsey, J., 2002. 4-MTA: A new synthetic drug on the dance scene. Drug Alcohol Depend 67, 111-115.

Zimmermann, U.S., Winkelmann, P.R., Pilhatsch, M., Nees, J.A., Spanagel, R., Schulz, K., 2009. Withdrawal phenomena and dependence syndrome after the consumption of "spice gold". Deutsches Arzteblatt 106, 464-467. 


\section{Figure Captions}

Figure 1. Frequency of synthetic and natural cannabis use among recent users of both drug types $(N=837)$

Figure 2. Speed of onset, time to peak effect, and duration of peak effect for synthetic and natural cannabis 